\title{
Platelet Indices in Colorectal Cancer Patients with Synchronous Liver Metastases
}

\author{
Li Li, ${ }^{1}$ Xiao-Yi Huang, ${ }^{2} \mathrm{Na} \mathrm{Li}^{3}{ }^{3}$ Ming-ming Cui, ${ }^{3}$ and Rui-tao Wang ${ }^{3}{ }^{3}$ \\ ${ }^{1}$ Department of Colorectal Surgery, Harbin Medical University Cancer Hospital, Harbin Medical University, Harbin, \\ Heilongjiang 150081, China \\ ${ }^{2}$ Biotherapy Center, Harbin Medical University Cancer Hospital, Harbin Medical University, Harbin, Heilongjiang 150081, China \\ ${ }^{3}$ Department of Internal Medicine, Harbin Medical University Cancer Hospital, Harbin Medical University, Harbin, \\ Heilongjiang 150081, China \\ Correspondence should be addressed to Rui-tao Wang; ruitaowang@126.com
}

Received 14 July 2019; Revised 21 September 2019; Accepted 15 October 2019; Published 4 November 2019

Academic Editor: Tatsuya Toyokawa

Copyright (C) $2019 \mathrm{Li} \mathrm{Li}$ et al. This is an open access article distributed under the Creative Commons Attribution License, which permits unrestricted use, distribution, and reproduction in any medium, provided the original work is properly cited.

\begin{abstract}
Aims. Liver metastases occur in approximately $25 \%$ of colorectal cancer (CRC) patients and cause more than $90 \%$ of deaths in CRC. Platelets play a crucial role in cancer progression and metastases. We aimed to investigate the relationship between platelet indices and CRC with synchronous liver metastases. Methods. We conducted a retrospective clinical study including 206 CRC patients without metastases and 200 CRC patients with synchronous liver metastases from January 1, 2015, to December 31, 2017. Data of the patients' clinicopathological characteristics were collected. Results. Platelet distribution width (PDW) was decreased in CRC patients with liver metastases compared with CRC patients without liver metastases. In addition, the prevalence of liver metastases reduced as PDW quartiles increased. After adjusting for other risk factors, the odds ratios (95\% confidence intervals) for CRC liver metastases according to PDW quartiles were 1.000, 0.289 (0.156-0.535), 0.482 (0.271-0.860), and 0.190 (0.101-0.358). Conclusions. Compared with CRC patients without metastases, PDW is reduced in CRC patients with liver metastases. Moreover, PDW was independently associated with the presence of CRC liver metastases.
\end{abstract}

\section{Introduction}

Liver metastases occur in approximately $25 \%$ of colorectal cancer (CRC) patients and cause more than $90 \%$ of deaths in CRC [1]. CRC patients with liver metastases have an estimated 5-year survival of 38\% [2]. Therefore, identification of novel serum biomarkers for CRC with liver metastases is urgently needed.

Platelets act as a crucial role in tumor growth and metastasis [3, 4]. Mean platelet volume (MPV) reflects platelet size and indicates platelet activation in clinical practice [5]. Platelet distribution width (PDW) reflects variation in platelet size and differentiates thrombocytopenia [6]. MPV was found to be altered in numerous tumors, such as lung, breast, gastric, ovarian, and colorectal cancers [7-11]. Our previous study also revealed that elevated MPV predicts a worse prognosis in CRC patients [12]. However, there is no study investigating the clinical implications of platelet indices in metastatic CRC patients.

We aimed to evaluate the relationship between platelet indices and CRC with liver metastases.

\section{Methods}

2.1. Study population. From January 2015 to December 2017, 200 CRC patients with synchronous liver metastases and 206 CRC patients without metastases at the Harbin Medical University Cancer Hospital were included in this study. CRC was histologically diagnosed. CRC synchronous liver metastases were defined as liver metastases detected at or before diagnosis of CRC [13]. Liver metastases were assessed with liver magnetic resonance imaging (MRI). All patients 
TABLE 1: Baseline characteristics of CRC patients.

\begin{tabular}{|c|c|c|c|}
\hline Variables & With liver metastases & Without liver metastases & $P$ value \\
\hline$N$ & 200 & 206 & \\
\hline Age (years) & $59.0 \pm 11.4$ & $58.2 \pm 10.0$ & 0.423 \\
\hline Gender (male, \%) & $125(62.5)$ & $111(53.9)$ & 0.079 \\
\hline $\mathrm{BMI}\left(\mathrm{kg} / \mathrm{m}^{2}\right)$ & $23.5 \pm 3.0$ & $23.6 \pm 3.4$ & 0.936 \\
\hline \multicolumn{4}{|l|}{ Smoker $(n, \%)$} \\
\hline Ever/current (\%) & $54(27.0)$ & $47(22.8)$ & 0.330 \\
\hline \multicolumn{4}{|l|}{ Drinking status } \\
\hline Ever/current (\%) & $45(22.5)$ & $49(23.8)$ & 0.759 \\
\hline $\mathrm{FPG}(\mathrm{mmol} / \mathrm{L})$ & $5.20(4.78-5.90)$ & $5.12(4.73-5.46)$ & 0.141 \\
\hline Albumin (g/L) & $41.3 \pm 6.7$ & $43.5 \pm 7.0$ & 0.001 \\
\hline Haemoglobin (g/dL) & $121.1 \pm 24.4$ & $128.8 \pm 23.7$ & 0.001 \\
\hline $\mathrm{WBC}\left(\times 10^{9} / \mathrm{L}\right)$ & $7.6 \pm 2.6$ & $7.0 \pm 2.6$ & 0.012 \\
\hline Platelet count $\left(\times 10^{9} / \mathrm{L}\right)$ & $288.3 \pm 102.5$ & $271.8 \pm 100.2$ & 0.102 \\
\hline MPV (fL) & $8.5 \pm 1.0$ & $8.3 \pm 1.4$ & 0.299 \\
\hline PDW (\%) & $16.9 \pm 0.9$ & $17.4 \pm 1.0$ & $<0.001$ \\
\hline Primary tumor location & & & 0.096 \\
\hline Colon & $139(69.5)$ & $127(61.7)$ & \\
\hline Rectum & $61(30.5)$ & $79(38.3)$ & \\
\hline $\mathrm{T}$ classification & & & 0.006 \\
\hline $\mathrm{T} 1+\mathrm{T} 2$ & $21(10.5)$ & $42(20.4)$ & \\
\hline $\mathrm{T} 3+\mathrm{T} 4$ & $179(89.5)$ & $164(79.6)$ & \\
\hline Lymph node metastasis & & & 0.006 \\
\hline Absent & $71(35.5)$ & $101(49.0)$ & \\
\hline Present & $129(64.5)$ & $105(51.0)$ & \\
\hline Differentiation & & & 0.462 \\
\hline Poor & $48(72.8)$ & $56(27.2)$ & \\
\hline Well/moderate & $152(24.0)$ & $150(72.8)$ & \\
\hline
\end{tabular}

CRC: colorectal cancer; FPG: fasting plasma glucose; WBC: white blood cell; BMI: body mass index; MPV: mean platelet volume; PDW: platelet distribution width.

received MRI scanning of the head, CT lung screening, liver MRI, and ${ }^{99 \mathrm{~m}}$ Tc-MDP bone scan. The exclusion criteria were the following: (1) underwent radiotherapy or chemotherapy prior to the enrolment, (2) had hematological disorders, (3) administration of acetylic salicylic acid, and (4) had other organ metastasis besides the liver.

The study was approved by the institutional review boards of Harbin Medical University Cancer Hospital. The informed consent was waived because this was a retrospective study.

2.2. Statistical Analysis. Student's t-test (for continuous variables with normal distribution), Mann-Whitney $U$ test (for continuous variables with nonnormal distribution), and $\chi^{2}$ test (for categorical variables) were used to analyze the differences between two groups. Multivariate logistic regression analysis was used to calculate the odds ratios and 95\% confidence intervals for liver metastasis adjusting for other confounding factors. $P<0.05$ was considered statistically significant. All analyses were performed by using SPSS Statistics version 22.0 (SPSS Inc., Chicago, IL, USA).

\section{Results}

Table 1 summarizes the clinicopathological characteristics of CRC patients. Of the 406 CRC patients enrolled, 236 (58.1\%) were men and $170(41.9 \%)$ were women. The patients with liver metastasis had a lower albumin, haemoglobin, and PDW and higher white blood cell counts. The number of patients with poor differentiation was 48 and 56 in the metastasis and nonmetastasis groups, respectively. However, statistical significances were not found in age, gender, smoking status, drinking status, fasting plasma glucose, platelet count, and MPV levels between two groups.

The platelet indices in CRC patients were shown in Table 2 and Table 3. In the group of nonliver metastasis, platelet count was significantly associated with $\mathrm{T}$ stage. MPV was associated with tumor size and PDW with lymph node metastasis. In the group with liver metastasis, we failed to observe the associations between platelet indices and primary tumor location, tumor size, differentiation, $\mathrm{T}$ classification, lymph node metastasis, metastatic tumor size, and metastatic tumor nodules. 
TABLE 2: Platelet indices in CRC patients without liver metastases.

\begin{tabular}{|c|c|c|c|c|c|c|}
\hline Variables & PLT & $P$ value & MPV & $P$ value & PDW & $P$ value \\
\hline Primary tumor location & & 0.005 & & 0.905 & & 0.605 \\
\hline Colon & $298.1(123.1)$ & & $8.4(1.7)$ & & $17.4(1.0)$ & \\
\hline Rectum & $254.5(77.4)$ & & $8.3(1.2)$ & & $17.3(1.0)$ & \\
\hline Tumor size $(\mathrm{cm})$ & & 0.487 & & 0.001 & & 0.165 \\
\hline$<5.0$ & $275.2(104.9)$ & & $8.1(1.2)$ & & $17.3(0.9)$ & \\
\hline$\geq 5.0$ & $264.8(89.8)$ & & $8.8(1.7)$ & & $17.5(1.1)$ & \\
\hline Differentiation & & 0.279 & & 0.206 & & 0.698 \\
\hline Well/moderate & $267.2(94.1)$ & & $8.4(1.4)$ & & $17.4(1.0)$ & \\
\hline Poor & $284.2(115.0)$ & & $8.1(1.4)$ & & $17.3(0.9)$ & \\
\hline $\mathrm{T}$ classification & & 0.035 & & 0.084 & & 0.526 \\
\hline $\mathrm{T} 1+\mathrm{T} 2$ & $242.8(88.8)$ & & $8.7(1.1)$ & & $17.4(1.1)$ & \\
\hline $\mathrm{T} 3+\mathrm{T} 4$ & $279.3(101.8)$ & & $8.3(1.5)$ & & $17.3(0.9)$ & \\
\hline Lymph node metastasis & & 0.461 & & 0.059 & & 0.034 \\
\hline Absent & $266.6(113.8)$ & & $8.5(1.3)$ & & $17.5(1.0)$ & \\
\hline Present & $276.9(85.3)$ & & $8.2(1.5)$ & & $17.2(0.8)$ & \\
\hline
\end{tabular}

PLT: platelet count; MPV: mean platelet volume; PDW: platelet distribution width.

TABle 3: Platelet indices in CRC patients with liver metastases.

\begin{tabular}{|c|c|c|c|c|c|c|}
\hline Variables & PLT & $P$ value & MPV & $P$ value & PDW & $P$ value \\
\hline Primary tumor location & & 0.251 & & 0.614 & & 0.778 \\
\hline Colon & $293.8(95.8)$ & & $8.5(1.0)$ & & $16.9(1.0)$ & \\
\hline Rectum & $275.8(116.2)$ & & $8.5(1.0)$ & & $16.9(0.8)$ & \\
\hline Tumor size $(\mathrm{cm})$ & & 0.815 & & 0.520 & & 0.811 \\
\hline$<5.0$ & $286.5(111.8)$ & & $8.5(1.1)$ & & $16.9(1.0)$ & \\
\hline$\geq 5.0$ & $289.9(93.9)$ & & $8.4(1.0)$ & & $16.9(1.0)$ & \\
\hline Differentiation & & 0.476 & & 0.378 & & 0.600 \\
\hline Poor & $275.5(102.4)$ & & $8.3(0.8)$ & & $16.8(0.8)$ & \\
\hline Well/moderate & $290.4(102.6)$ & & $8.5(1.1)$ & & $16.9(0.9)$ & \\
\hline $\mathrm{T}$ classification & & 0.956 & & 0.551 & & 0.958 \\
\hline $\mathrm{T} 1+\mathrm{T} 2$ & $287.1(95.9)$ & & $8.3(1.4)$ & & $16.9(0.7)$ & \\
\hline $\mathrm{T} 3+\mathrm{T} 4$ & $288.5(103.5)$ & & $8.5(1.0)$ & & $16.9(0.9)$ & \\
\hline Lymph node metastasis & & 0.660 & & 0.852 & & 0.313 \\
\hline Absent & $284.0(84.1)$ & & $8.5(0.9)$ & & $17.0(0.9)$ & \\
\hline Present & $290.7(115.5)$ & & $8.5(1.1)$ & & $16.9(0.9)$ & \\
\hline Metastatic tumor size $(\mathrm{cm})$ & & 0.815 & & 0.520 & & 0.811 \\
\hline$<5.0$ & $286.5(111.8)$ & & $8.5(1.1)$ & & $16.9(0.9)$ & \\
\hline$\geq 5.0$ & $289.9(93.9)$ & & $8.4(1.0)$ & & $16.9(1.0)$ & \\
\hline Metastatic tumor nodules & & 0.420 & & 0.134 & & 0.979 \\
\hline$<2$ & $300.4(133.1)$ & & $8.3(1.0)$ & & $16.9(0.8)$ & \\
\hline$\geq 2$ & $285.5(94.1)$ & & $8.5(1.0)$ & & $16.9(1.0)$ & \\
\hline
\end{tabular}

PLT: platelet count; MPV: mean platelet volume; PDW: platelet distribution width.

The ROC curve for metastasis was used to verify the optimum cutoff points for PDW. A PDW $\leq 16.5 \%$ was shown to be predictive for CRC liver metastasis, with a sensitivity of $51.0 \%$ and a specificity of $85.2 \%$ (area under the curve $0.712 ; 95 \%$ confidence interval $0.659-0.766 ; P<0.001$ ).

The prevalence of liver metastasis was calculated by the quartiles of PDW levels (Figure 1). The prevalence rate of liver metastasis in quartile 1 , quartile 2 , quartile 3 , and quartile 4 was $70.1 \%$ (82/117), $41.6 \%$ (37/89), $50.5 \%$ (54/107), and 29.0\% (27/93), respectively.

The risks of liver metastasis according to PDW quartiles are analyzed and shown in Table 4 . After adjusting for age, gender, BMI, smoking status, drinking status, WBC, haemoglobin, albumin, and fasting plasma glucose, the prevalence 


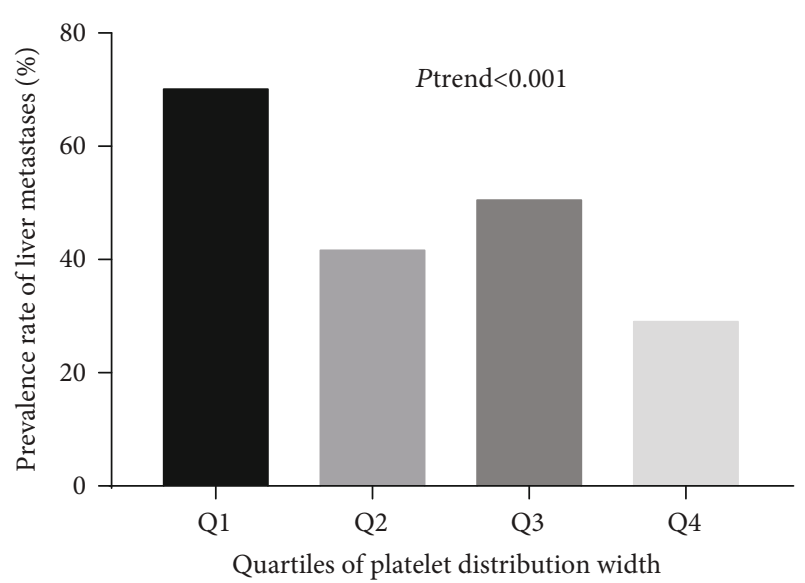

FIGURE 1: The prevalence of liver metastasis in CRC patients according to the quartiles of PDW levels.

TABLE 4: The risks of liver metastases in CRC patients according to PDW quartiles.

\begin{tabular}{lcccc}
\hline & Cases & Controls & OR $(95 \% \mathrm{CI})$ & $P$ value \\
\hline Q1 $(\leq 16.5 \%)$ & 82 & 35 & 1 (reference) & \\
Q2 $(16.6-17.0 \%)$ & 37 & 52 & $0.289(0.156-0.535)$ & $<0.001$ \\
Q3 $(17.1-17.7 \%)$ & 54 & 53 & $0.482(0.271-0.860)$ & 0.013 \\
Q4 ( $\geq 17.8 \%)$ & 27 & 66 & $0.190(0.101-0.358)$ & $<0.001$ \\
\hline
\end{tabular}

Logistic regression analysis adjusted for age, gender, BMI, smoking status, drinking status, albumin, FPG, haemoglobin, and WBC. CI: confidence interval.

risk of liver metastasis for the highest quartile of PDW was 0.190 (0.101-0.358).

\section{Discussion}

Our study demonstrated that the CRC patients with liver metastases have lower PDW levels compared to those without metastases and PDW was independently associated with the presence of liver metastases.

The interaction of tumor cells with platelets leads to platelet activation, which in turn promotes tumor progression and metastasis [14]. Elevated platelet-derived growth factor D promotes CRC cell proliferation and invasion by upregulating the expression of Notch 1 and matrix metalloproteinase 9 [15]. Moreover, platelet-derived endothelial cell growth factor levels were increased in CRC patients and were associated with poor prognosis $[16,17]$. A recent study confirmed that serum platelet-derived growth factor AA is an independent predictor for CRC liver metastasis [18]. In addition, the use of low-dose aspirin could restore antitumor activity by inhibiting platelet COX-1 [19]. Our study confirmed the important role of platelet activation in CRC. Moreover, our results provide the basis for applying antiplatelet therapy in CRC patients with liver metastases.

The mechanisms underlying the association between reduced PDW and CRC liver metastases remain unclear. The interaction between platelets and tumor cells induces cell plasticity and promotes cancer metastasis by enhancing circulating tumor cell survival and extravasation [20]. PDW is an indicator of the average change in platelet volume. Platelet volume is determined both during megakaryopoiesis and thrombopoiesis. The decrease of PDW reflects the failure of heterogenic megakaryocytic maturation [21]. The thrombocytopoiesis is regulated by many factors, among which the key factor has been attributed to thrombopoietin (TPO) [22]. Elevated plasma TPO levels have been observed in cancer patients with advanced stage [23]. Recent study found that PDW was a better indicator to reflect the characteristics of activated platelets [24]. The secretory factors released by activated platelets promote the expression of cytokines, proteolytic enzymes, and chemokines within the microenvironment and accelerate cancer invasion [25]. Overexpression of platelet-derived growth factor (PDGF) was related to uncontrolled angiogenesis in CRC patients with liver metastases and was found to be a new prognostic indicator for a worse prognosis in CRC $[18,26]$. In addition, overexpression of PDGF receptor is associated with advanced stage disease in stromal cells of human colon carcinomas [27]. In addition, recent studies revealed that dual antiplatelet therapy (aspirin and clopidogrel) inhibits the expression of $\alpha$-granule-stored proteins and decreases the heterotypic interactions between platelets/leukocytes and the endothelium [28].

Our current study bears several limitations. Firstly, it was a retrospective and single center study. Secondly, the mechanisms underlying the association are needed to clarify. Thirdly, the conclusion could not be applied to other ethnic groups because the cohorts in our study were composed of Chinese patients.

In summary, compared with CRC patients without metastases, PDW is reduced in CRC patients with liver metastases. Moreover, PDW was independently associated with the presence of CRC liver metastases.

\section{Data Availability}

The data used to support the findings of this study are available from the corresponding authors upon request.

\section{Ethical Approval}

The study was approved by the institutional review boards of Harbin Medical University Cancer Hospital.

\section{Conflicts of Interest}

The authors declare that they have no conflicts of interest.

\section{Authors' Contributions}

RT W conceived the study; L L, XY H, and RT W participated in the design; L L, XY H, N L, and MM C collected the data; and $\mathrm{L} \mathrm{L}, \mathrm{XY} \mathrm{H}, \mathrm{N} \mathrm{L}$, and MM C performed the statistical analyses. L L and XY $\mathrm{H}$ drafted the manuscript. N L, MM $\mathrm{C}$, and RT W edited and checked the manuscript. All of the authors have read and approved the final manuscript. $\mathrm{Li} \mathrm{Li}$ and Xiao-Yi Huang contributed equally to this work. 


\section{Acknowledgments}

This study was jointly supported by the Haiyan Foundation of Harbin Medical University Cancer Hospital (JJZD2017-05), the Nn10 Program of Harbin Medical University Cancer Hospital, and the Innovative Ability Promotion Program of Heilongjiang Province for Scientific Research Institutions (YC2016D002).

\section{References}

[1] J. Engstrand, H. Nilsson, C. Strömberg, E. Jonas, and J. Freedman, "Colorectal cancer liver metastases - a population-based study on incidence, management and survival," BMC Cancer, vol. 18, no. 1, p. 78, 2018.

[2] G. P. Kanas, A. Taylor, J. N. Primrose et al., "Survival after liver resection in metastatic colorectal cancer: review and metaanalysis of prognostic factors," Clinical Epidemiology, vol. 4, pp. 283-301, 2012.

[3] N. M. Bambace and C. E. Holmes, "The platelet contribution to cancer progression," Journal of Thrombosis and Haemostasis, vol. 9, no. 2, pp. 237-249, 2011.

[4] H. A. Goubran, J. Stakiw, M. Radosevic, and T. Burnouf, "Platelet-cancer interactions," Seminars in Thrombosis and Hemostasis, vol. 40, no. 3, pp. 296-305, 2014.

[5] C. DeSantis, J. Ma, L. Bryan, and A. Jemal, "Breast cancer statistics, 2013," CA: A Cancer Journal for Clinicians, vol. 64, no. 1, pp. 52-62, 2014.

[6] K. Kaito, H. Otsubo, N. Usui et al., "Platelet size deviation width, platelet large cell ratio, and mean platelet volume have sufficient sensitivity and specificity in the diagnosis of immune thrombocytopenia," British Journal of Haematology, vol. 128, no. 5, pp. 698-702, 2005.

[7] Y. Kemal, G. Demirağ, K. Ekiz, and I. Yücel, "Mean platelet volume could be a useful biomarker for monitoring epithelial ovarian cancer," Journal of Obstetrics and Gynaecology, vol. 34, no. 6, pp. 515-518, 2014.

[8] S. Kumagai, J. Tokuno, Y. Ueda et al., "Prognostic significance of preoperative mean platelet volume in resected non-smallcell lung cancer," Molecular and Clinical Oncology, vol. 3, no. 1, pp. 197-201, 2015.

[9] X. M. Shen, Y. Y. Xia, L. Lian et al., "Mean platelet volume provides beneficial diagnostic and prognostic information for patients with resectable gastric cancer," Oncology Letters, vol. 12, no. 4, pp. 2501-2506, 2016.

[10] O. Tanriverdi, S. Menekse, F. Teker et al., "The mean platelet volume may predict the development of isolated bone metastases in patients with breast cancer: a retrospective study of the Young Researchers Committee of the Turkish Oncology Group (TOG)," Journal of BUON, vol. 21, no. 4, pp. 840850, 2016.

[11] J. Y. Li, Y. Li, Z. Jiang, R. T. Wang, and X. S. Wang, "Elevated mean platelet volume is associated with presence of colon cancer," Asian Pacific Journal of Cancer Prevention, vol. 15, no. 23, pp. 10501-10504, 2015.

[12] M. M. Cui, N. Li, X. Liu et al., "Platelet distribution width correlates with prognosis of non-small cell lung cancer," Scientific Reports, vol. 7, no. 1, p. 3456, 2017.

[13] R. Adam, A. de Gramont, J. Figueras et al., "Managing synchronous liver metastases from colorectal cancer: a multi- disciplinary international consensus," Cancer Treatment Reviews, vol. 41, no. 9, pp. 729-741, 2015.

[14] P. Gresele, S. Momi, M. Malvestiti, and M. Sebastiano, "Platelet-targeted pharmacologic treatments as anti-cancer therapy," Cancer Metastasis Reviews, vol. 36, no. 2, pp. 331-355, 2017.

[15] B. Jiang, J. Chen, W. Yuan et al., "Platelet-derived growth factor-D promotes colorectal cancer cell migration, invasion and proliferation by regulating Notch 1 and matrix metalloproteinase-9," Oncology Letters, vol. 15, no. 2, pp. 1573-1579, 2018.

[16] M. Matsumura, Y. Chiba, C. Lu et al., "Platelet-derived endothelial cell growth factor/thymidine phosphorylase expression correlated with tumor angiogenesis and macrophage infiltration in colorectal cancer," Cancer Letters, vol. 128, no. 1, pp. 55-63, 1998.

[17] T. Tsuji, T. Sawai, H. Yamashita et al., "Platelet-derived endothelial cell growth factor expression is an independent prognostic factor in colorectal cancer patients after curative surgery," European Journal of Surgical Oncology, vol. 30, no. 3, pp. 296-302, 2004.

[18] H. D. Pan, Y. F. Peng, G. Xiao, and J. Gu, "High levels of serum platelet-derived growth factor-AA and human epidermal growth factor receptor-2 are predictors of colorectal cancer liver metastasis," World Journal of Gastroenterology, vol. 23, no. 7, pp. 1233-1240, 2017.

[19] M. G. Sciulli, P. Filabozzi, S. Tacconelli et al., "Platelet activation in patients with colorectal cancer," Prostaglandins, Leukotrienes, and Essential Fatty Acids, vol. 72, no. 2, pp. 79-83, 2005.

[20] S. Wang, Z. Li, and R. Xu, "Human cancer and platelet interaction, a potential therapeutic target," International Journal of Molecular Sciences, vol. 19, no. 4, p. 1246, 2018.

[21] K. Matsuo, S. H. Tang, B. Sharifi, S. A. Rubin, R. Schreck, and J. A. Fagin, "Growth factor production by human thyroid carcinoma cells: abundant expression of a platelet-derived growth factor-B-like protein by a human papillary carcinoma cell line," The Journal of Clinical Endocrinology and Metabolism, vol. 77, no. 4, pp. 996-1004, 1993.

[22] V. Dymicka-Piekarska and H. Kemona, "Thrombopoietin and reticulated platelets as thrombopoietic markers in colorectal cancer," Thrombosis Research, vol. 122, no. 1, pp. 141-143, 2008.

[23] Y. Sasaki, T. Takahashi, H. Miyazaki et al., "Production of thrombopoietin by human carcinomas and its novel isoforms," Blood, vol. 94, no. 6, pp. 1952-1960, 1999.

[24] A. Leader, D. Pereg, and M. Lishner, "Are platelet volume indices of clinical use? A multidisciplinary review," Annals of Medicine, vol. 44, no. 8, pp. 805-816, 2012.

[25] B. Tesfamariam, "Involvement of platelets in tumor cell metastasis," Pharmacology \& Therapeutics, vol. 157, pp. 112-119, 2016.

[26] Y. Nakamura, F. Tanaka, Y. Yoshikawa et al., "PDGF-BB is a novel prognostic factor in colorectal cancer," Annals of Surgical Oncology, vol. 15, no. 8, pp. 2129-2136, 2008.

[27] Y. Kitadai, T. Sasaki, T. Kuwai et al., "Expression of activated platelet-derived growth factor receptor in stromal cells of human colon carcinomas is associated with metastatic potential," International Journal of Cancer, vol. 119, no. 11, pp. 2567-2574, 2006.

[28] A. K. Mandal and D. B. Mount, "The molecular physiology of uric acid homeostasis," Annual Review of Physiology, vol. 77, no. 1, pp. 323-345, 2015. 


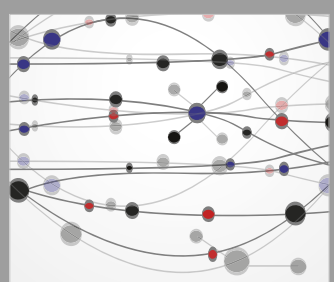

The Scientific World Journal
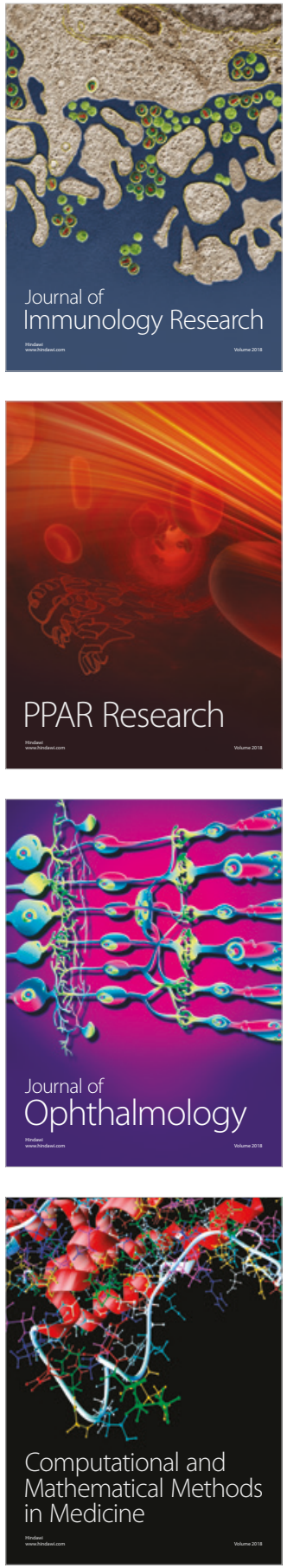

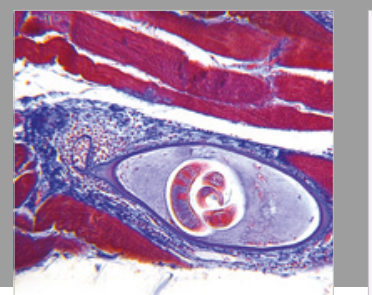

Gastroenterology Research and Practice

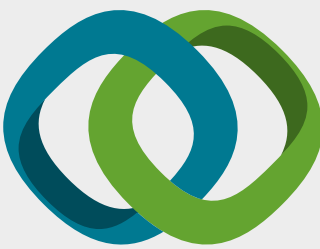

\section{Hindawi}

Submit your manuscripts at

www.hindawi.com
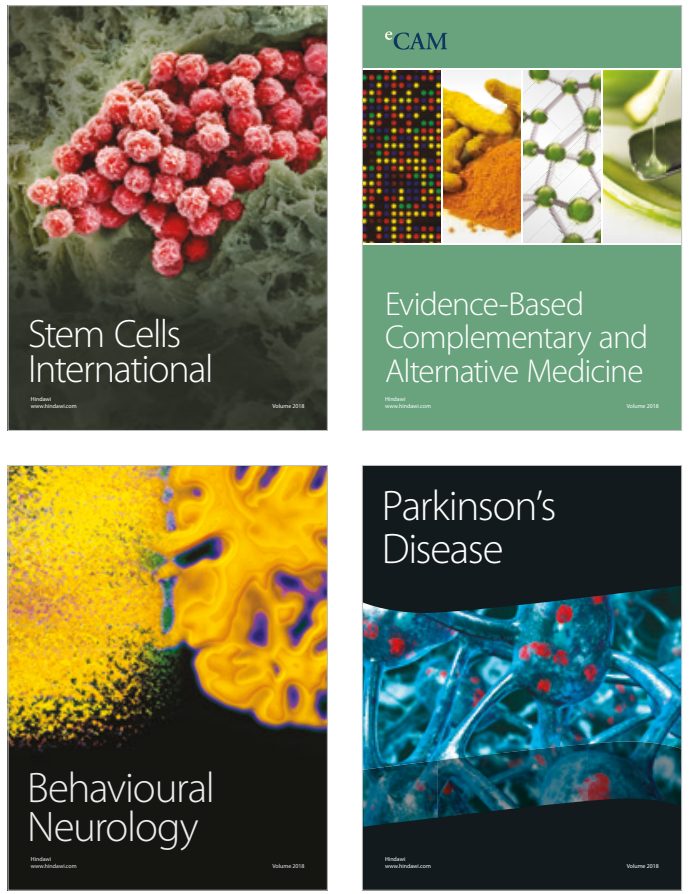

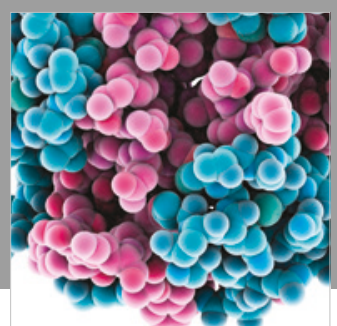

ournal of

Diabetes Research

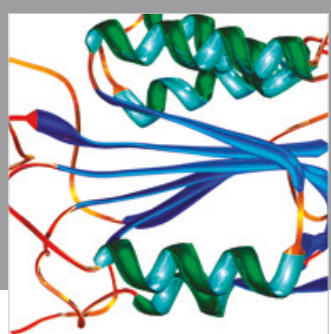

Disease Markers
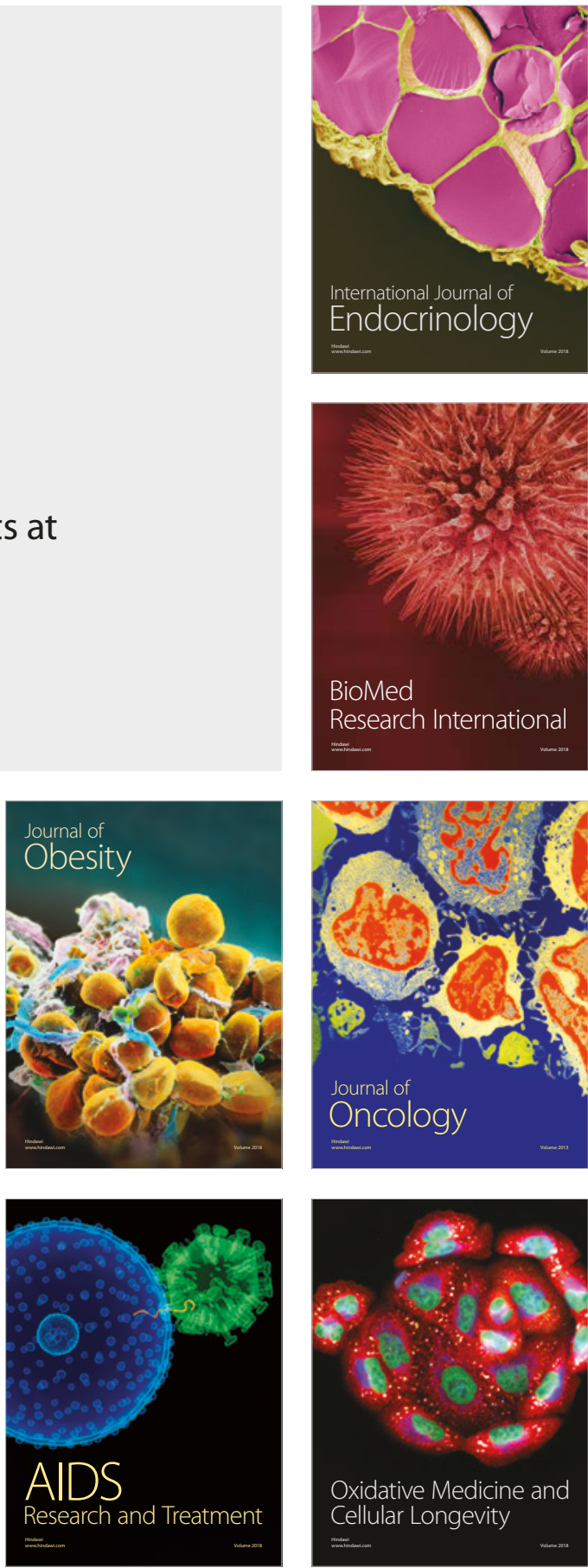